\title{
APORTACIONES DEL FEMINISMO AL DERECHO INTERNACIONAL EN LA CONSTRUCCIÓN DE LA PAZ
}

\author{
NATALIA ÁLVAREZ MOLINERO \\ Universidad de Aberdeen (Reino Unido)
}

\section{ALGUNAS VIRTUDES DEL FEMINISMO}

Feminismo y Derecho Internacional conforman un par relativamente reciente. A las mujeres que trabajamos en Derecho Internacional cuando hablamos de feminismo ${ }^{1}$ nos suelen remitir al apartado relativo a derechos de las mujeres. Sin embargo a estas alturas es fácil entender que se pueden defender los derechos de las mujeres y no ser feminista en absoluto. Una de las perspectivas que se puede adoptar respecto a los derechos de las mujeres es la de buscar proteger a este colectivo y equiparlo en derechos y oportunidades con los varones, al mismo tiempo que reconocerlo como víctima cuando sus derechos y libertades han sido vulnerados mientras se contextualiza como «un grupo vulnerable». Si bien es cierto que en los últimos años en Derecho Internacional ha existido un avance en lo relativo al reconocimiento de los derechos de las mujeres desde la perspectiva del empoderamiento, que aboga por maximizar la participación política de las mujeres en los espacios públicos, y en general por aumentar su participación en las esferas en las que las que se toman las decisiones, lo cierto

1. Somos conscientes de la existencia de diferentes feminismos que en Derecho Internacional se han hecho patentes, entre otros, en las distintas posiciones mantenidas por los grupos de mujeres en temas como el tráfico de mujeres o la prostitución. Cuando mencionamos el feminismo como categoría general en este artículo, nos referimos al espacio de debate en el que se articulan posturas aglutinadas entorno a la pregunta de cuáles son y cómo funcionan los mecanismos exclusión/ dominacion de las mujeres, y las consecuencias de la imposición de una visión dominantemente masculina en nuestras sociedades y en el derecho. 
es que estos derechos se han mostrado un tanto «líquidos» $»^{2}$ en lo que se refiere a su capacidad de reformar estructuras de forma permanente ${ }^{3}$.

Un ejemplo relativo a la dificultad de incidir en estereotipos y concepciones sexistas sobre la mujer puede darnos una idea sobre las barreras a las que nos enfrentamos. En Estados Unidos el Congreso aprobó una ley en 2004 por la que se establece que el feto es legalmente un ser independiente respecto a su madre, lo que significa que éste puede ser víctima de la una violación de sus derechos fundamentales en la que la agresora sea por su propia progenitora. En estos momentos, más de 20 estados en Estados Unidos han aprobado leyes por las que se permite iniciar procedimientos penales contra madres que durante su embarazo han llevado a cabo conductas consideradas peligrosas y que puedan dañar la salud del feto ${ }^{4}$. Las razones que algunos grupos esgrimen para adoptar estas medidas están relacionadas con el hecho de que en 2003 la mortalidad infantil se incrementó notablemente en Estados Unidos, siendo 3 veces más alta que la de Japón y 2,5 que la de Finlandia o Noruega. En función de estos datos, el gobierno federal elaboró unas líneas generales a adoptar por los sistemas sanitarios en los que se recomienda que todas las mujeres en edad fértil, tanto si están embarazadas como si no lo están, sean tratadas como pre-embarazadas. Esta idea se concreta en una serie de recomendaciones para toda mujer en edad fértil entre las que se encuentra tomar suplementos de ácido fólico, no fumar, mantener un peso adecuado y controlar las enfermedades crónicas como asma o diabetes ${ }^{5}$.

2. Bauman se refiere a la modernidad líquida en términos de su contingencia, su provisionalidad, una sociedad en la que todo tiene fecha de caducidad, en la que todo cambia permanentemente. Si la modernidad sólida nos invitaba a pensar en un futuro en el que se produciría un cambio estable y permanente, la modernidad líquida es la de la incertidumbre, la vulnerabilidad y la inseguridad. BAUmAn, Zygmunt: La modernidad líquida, Buenos Aires, Fondo de Cultura Económica, 2002. Algunas reivindicaciones relativas a los derechos de las mujeres se insertan en cierta manera en este esquema, si tenemos en cuenta que remedian situaciones concretas más que inciden en las causas estructurales de las mismas. Nos referimos por ejemplo al derecho a un igual salario por el mismo trabajo o al derecho a la salud reproductiva. Ambas reivindicaciones pueden sostenerse en marcos en los que un análisis de género no se tenga en cuenta. Así, se puede obtener un igual salario por el mismo trabajo, pero el acceso a las promociones se obtiene en función de las horas extras que las mujeres madres de familia no están dispuestas a realizar; o se reconoce el derecho al acceso a la planificación familiar, pero no se tienen en cuenta las diferencias culturales.

3. En uno de los informes presentados por el Alto Comisionado para los Derechos Humanos en relación con el tema de la pobreza y la participación política de las mujeres se afirma: «Una de las tendencias alarmantes que se destacan al examinar la aplicación del Programa de Hábitat es que las medidas jurídicas y políticas no están teniendo mucho efecto en la reducción de la brecha existente entre la pobreza de los hombres y la de las mujeres. Los prejuicios sexistas persistentes en todos los aspectos de la sociedad, en particular cuando se trata de abordar el problema de la pobreza, se basan en conceptos sociales vigentes desde hace tiempo.» ("La lucha contra la pobreza y el derecho a la participación: el papel de las mujeres. Nota de antecedentes preparada por la Oficina del Alto Comisionado para los Derechos Humanos», A/HCR/Sub.1/58/SF/2, 14 de junio de 2006, pp. 4-5).

4. Entre estas conductas se encuentra el hecho de que los médicos aconsejen una cesárea en el parto y la mujer se niegue a la citada intervención.

5. Washington Post, 16 de mayo de 2006. 
No deja de ser irónico el hecho de que después de tantos años de lucha para lograr que las mujeres no seamos únicamente consideradas por la sociedad como mujeres-madres, sino como seres independientes respecto a la circunstancia de la maternidad, resulta que nuestra independencia y nuestro derecho a elegir la maternidad como opción y no como imposición se traduce en que cuando elegimos ser madres se nos considera portadoras de vidas humanas, meras transportistas de embriones y fetos con responsabilidades legales si la mercancía no llega a su destino en las condiciones esperadas. Debe ser una virtud del patriarcado, su capacidad de transmutar los intereses y deseos de las mujeres en nuevas ataduras.

Si no queremos ser consideradas y valoradas únicamente como mujeresmadres y reivindicamos nuestra vida propia con o sin hijos/as, entonces, la sociedad establece que aquellas que deciden ser madres lo hacen por decisión propia, por devoción y pasión a la sociedad a la que desean contribuir engendrando futuros seres humanos sanos. Esas nuevas vidas constituyen la máxima contribución que una mujer puede hacer a la sociedad, su mejor producto, y por lo tanto merece que se ejerza sobre ella un control de calidad. En consecuencia, el ser, el rol, el sujeto que se protege es la madre, no la mujer, lo que nos demuestra que los estereotipos siguen funcionando de una forma extremadamente eficiente, pero tampoco lo olvidemos, de forma implacable y cruel.

Regina McKnight, ciudadana de Estados Unidos, joven, mujer afro-americana y con escasos recursos económicos, dio a luz en 1999 a un niño que nació muerto. Seis meses más tarde fue acusada de homicidio. Regina McKnight, desde que su madre murió atropellada, había comenzado a consumir drogas para paliar su depresión. Fue precisamente en ese período de tiempo en el que Regina se quedó embarazada y sin posibilidades de acceder a servicios de salud mental o de tratamiento de su adicción, ya que no estaban disponibles en su zona para personas sin recursos. En el juicio que se llevó a cabo, y a pesar de que los médicos no pudieron cerciorar que la muerte del niño se debiera a su consumo de cocaína, Regina fue condenada a 20 años de prisión, pena que posteriormente se rebajó a 12 años. Desgraciadamente, no es el único caso en Estados Unidos en el que una mujer ha sido acusada y encarcelada por negligencia durante su embarazo ${ }^{6}$.

Son muchas las consideraciones que podemos hacer en relación con este caso, una de ellas, no necesariamente la más importante, es la que nos sugiere que el hecho de traer una vida al mundo se sigue considerando una tarea exclusivamente femenina y de la que nosotras somos responsables. En este sentido, la contribución del varón al acto de la procreación es considerada en la mayoría de los casos apropiada, positiva, sana, deseable y puntual. Nadie cuestiona que los malos hábitos alimenticios, adicciones a sustancias diversas o enfermedades hereditarias o de orden genético puedan ser la causa de que un embarazo no

6. The Guardian, 4 de septiembre de 2006. Más información sobre este y otros casos puede obtenerse en www.advocatesforpregnanwomen.org. 
llegue a buen término. Tampoco se consideran factores medioambientales o de otro orden. Debido a que el acto del varón es perfecto en sí, e incuestionable, el cuerpo que hay que controlar, la persona a la que hay que castigar y la que en última instancia es responsable ante la sociedad y ante la ley es la mujer. Una segunda apreciación se refiere al hecho de que el proceso de debate de la ley, aprobación, aplicación, encausamiento de Regina y su final condena ha sido un proceso en el que hombres y mujeres han participado, y en especial mujeres que conforman grupos anti-abortistas o el movimiento por los derechos del feto.

Esto nos indica en primera instancia que la sociedad sigue entendiendo que determinadas tareas son exclusivamente femeninas, entre las que se incluye la maternidad y el cuidado del otro. La cuestión si embargo va más allá, no solamente se trata de que estas tareas se califiquen como femeninas, sino que debido a que son femeninas pueden ser objeto de escrutinio por parte del Estado. La sociedad ha hecho así sus ajustes. Si accedemos al espacio público, va a ser a costa en muchos casos de que nuestra esfera más íntima pueda ser vigilada y controlada. En segundo lugar, que las mujeres participemos en los procesos de toma de decisiones no asegura que se llegue a mejores resultados si las mujeres que alzan su voz son las más obedientes y adiestradas muestras de la eficiencia reproductiva del sistema patriarcal. Con ello no quiero decir que la participación de las mujeres no sea importante, sino que ésta, sin más, no asegura que se vayan a tomar decisiones que beneficien a las mujeres en general. La participación de las mujeres es irrenunciable como objetivo político, pero no asegura el fin de la discriminación como tal. Por ello estimo que el feminismo es especialmente relevante no sólo para los derechos humanos, sino también para el Derecho Internacional. El feminismo nos ayuda a revisar los entresijos, a entender cómo llegamos a esta situación, a desarrollar estrategias más allá del "yo tengo derecho"y en las que el protagonismo está en "yo deseo para mí». Esto es, traslada el debate de lo puramente jurídico y normativo a lo político-emocional en el que la realidad no se presenta como opciones excluyentes que obligan a legislar o en favor del feto o en favor de la mujer, a favor o en contra de la maternidad. El feminismo nos ayuda a navegar en estos escenarios tan complejos.

Esto me lleva a un segundo apunte que pretende desbancar la idea de que las mujeres somos víctimas y los hombres son verdugos. Que las mujeres nos encontremos en peores situaciones en términos de disfrute de derechos y reconocimiento de libertades en el mundo no quiere decir que ellos sean los únicos responsables. Esta idea nos ayuda a abrir más los ojos para ser conscientes de cómo operan los patrones de exclusión y dominación. No es posible instaurar un sistema de dominación y sujeción de las mujeres en el que nosotras no hayamos participado de alguna manera ${ }^{7}$. No se trata de una guerra entre sexos,

7. No se trata de culparnos, sino de hacernos conscientes del hecho de que el patriarcado ha supuesto y sigue suponiendo una opción atractiva y deseable para algunas mujeres, invisible para otras, tentadora para otras almas e irrelevantes para varias. Cualquier sistema complejo de dominación tiene varias zonas oscuras y numerosos aliados. Baudrillard nos recuerda como el deseo y su libe- 
no hay que vencer a ningún enemigo, hay que crear espacios de entendimiento, a veces desde la lucha, otras veces desde el diálogo. Si las mujeres nos posicionamos solamente como sujetos de derechos tenemos que ser conscientes que la lucha permanente por nuestros derechos fundamentales puede reducirnos como seres humanos a un papel de meros combatientes ${ }^{8}$.

En este escenario en el que las mujeres buscamos encontrar un lugar, el feminismo se interesa no sólo por los derechos sino también por los espacios en los que éste se ejerce y la manera en la que se desarrolla, al mismo tiempo que por los sujetos a los que excluye. El feminismo va más allá del mero derecho que se ejercita o se reclama, porque es una cuestión de poder. Sabemos que el poder funciona, que se mueve y no reside, y que actúa no directamente sobre las personas, sino sobre sus acciones. En una relación de poder, el otro/la otra es indispensable y reconocida al mismo tiempo como un sujeto libre que actúa. Por esa razón las relaciones de poder no son una confrontación cara a cara, sino una permanente provocación'. El feminismo se inscribe por lo tanto en esta lucha por desvelar las acciones a las que el sujeto, en este caso las mujeres, son compelidas como parte de ciertas estrategias de poder. Si las relaciones de poder nos sitúan ante la provocación, el feminismo nos rescata mediante la trasgresión. Sin lugar a dudas, no es una tarea fácil.

\section{LA INTERSECCIÓN FEMINISMO - DERECHO INTERNACIONAL}

Uno de los problemas iniciales con el que nos encontramos en la aplicación del feminismo como herramienta crítica en relación con el Derecho Internacional es que se nos convoca únicamente a intervenir en ciertos temas que se entienden como la única intersección posible entre ambas disciplinas. La ilusión que comparten muchos y muchas es que el Derecho Internacional se sitúa por encima del género, es un derecho que regula las relaciones entre Estados, las transacciones comerciales, los standards que se aplican en conflictos armados, en definitiva un derecho en el que el género parece que no tiene lugar porque se desdibuja en una serie de escenarios en los que incluso las personas humanas parecen subsumidas y sometidas a las estructuras estatales. En este escenario la contribución del feminismo es una contribución en términos de derechos de las mujeres y no de reparto del poder en el mundo.

Orford señala que existe una cierta corriente de feminismo que preconiza que las feministas sólo podemos contribuir al Derecho Internacional en dos esferas. Una primera sería aquella que sostiene que las académicas del mundo del derecho, y que vivimos en los países industrializados, deberíamos dedicarnos a

ración entra en esta categoría: "(..) si hasta aquí se había enseñado a las mujeres a no pedir nada para conducirlas a no desear nada, ¿no se les enseña hoy a pedirlo todo para no desear nada?» (BAUdrillard, Jean: De la seducción, Madrid, Cátedra, 2000, p. 29).

8. Collins, Francoise: "The praxis of difference», en Christina Howells (ed): French Women Philosophers: A Contemporary Reader, London, Routledge, 2004, p. 16.

9. Foucault, Michel: "The subject and the power», en D. James Faubion (ed): Power: Essentials works of Foucault 1954-1984, vol III, New York, New York Press, 2000, pp. 340-342. 
recopilar información y producir un conocimiento sustancial sobre las mujeres víctimas de violaciones de derechos humanos en países del llamado tercer mundo. La segunda contribución posible vendría de la mano del análisis del diseño de normas y standards jurídicos en el ámbito de derechos de la mujer, derechos humanos y derecho penal internacional. Orford tilda ambas corrientes, siguiendo a Spivak, como formas de feminismo que contribuyen al imperialismo. La primera porque incita a que la producción y reproducción de conocimiento sobre el sufrimiento de las mujeres del tercer mundo se convierta en un objeto en sí mismo, más que en sujeto ${ }^{10}$. No es que Orford estime que éste sea un campo desdeñable, pero lo que parece cierto es que por mucho que queramos representar a las mujeres oprimidas del sur y a sus demandas, no somos ellas. No podemos construirlas como sujetos que hablan sin quitarles la voz al mismo tiempo. "Hablar de», no es lo mismo que "hablar con», o "hablar por» (para esto último necesitamos una autorización) En el segundo caso, reducir la contribución del feminismo a los derechos humanos y al derecho penal internacional significa no tener en cuenta el potencial liberador y crítico del feminismo en todo lo relativo a las estructuras de poder. Estas asunciones implican al menos algunas aclaraciones de partida.

En primer lugar que el feminismo en Derecho Internacional puede y debe ir más allá de los derechos de las mujeres, de forma que las cuestiones que nos afectan a las mujeres no son sólo cuestiones de derechos. Ello implica ser consciente de la existencia de otras esferas de trabajo igualmente relevantes para las mujeres. Por ejemplo, ¿cómo nos afecta a las mujeres y qué importancia tiene el hecho de que las decisiones sobre el uso de la fuerza en Derecho Internacional se tomen por instancias mayoritariamente masculinas? ¿Cuáles son las razones por las que hasta mediados de los noventa no existieron decisiones de tribunales internacionales como el de la Ex Yugoslavia o Ruanda que emitieran sentencias contra individuos en que éstos fueran acusados de crímenes contra la humanidad por violaciones a mujeres?

En segundo lugar, ser conscientes de que la dicotomía publico-privado opera no sólo como esfera que invisibiliza aquello que no se quiere ver, a saber, lo público implica reconocimiento como individuo y como miembro de una comunidad, posibilidad de acción y participación, el lugar en el que las batallas se desarrollan, donde se consiguen los éxitos y los fracasos. Lo privado por su parte se adentra en lo doméstico, en lo frágil, en lo prescindible por no tener valor, lo penetrable y vulnerable, lo íntimo que debe ser protegido. Esta división colocó a las mujeres frente a una barrera infranqueable en la que o elegían carecer de valor como parte de lo que significaba ser "doméstica y privada» o perdían toda posibilidad de privacidad en virtud de su intromisión en lo público ${ }^{11}$. Sin

10. ORFORD, Anne: «Feminism, Imperialism and the Mission of International Law», Nordic Journal of International Law, 71 (2002), pp. 279-281.

11. Las mujeres no han sido las únicas destinatarias de esta dicotomía. En este sentido, Iris Marion Young afirma: «Incluso "padres» republicanos como Jefferson identificaron a los pieles rojas y los negros en sus territorios con la pasión y la naturaleza salvaje, de forma semejante al temor 
embargo, esta percepción ha sido también transferida a otras esferas y ámbitos del Derecho Internacional en los que la acción se prioriza sobre cualquier otra dinámica que pueda provenir de esferas consideradas privadas. A modo de ejemplo, cuando tratamos el tema de la intervención humanitaria existe una tendencia en esta disciplina a entender que cuando existen violaciones masivas de derechos humanos la opción más acertada es una intervención humanitaria de carácter armado (una acción) Lo opuesto a todo aquello que desafíe la idea de actuar en un momento determinado, incluso que cuestione el hecho que determinadas acciones puedan ser denominadas "humanitarias», es considerado inefectivo, improductivo y negativo ${ }^{12}$.

Las narrativas humanitarias ofrecen también un excelente ejemplo de cómo el mundo se nos presenta en categorías de dentro-fuera, salvadores-salvados, poderosos-inermes. Con frecuencia la comunidad internacional y los Estados ponen en marcha este tipo de narraciones para justificar acciones e intervenciones en otros países. Así la comunidad internacional juega el papel masculino de héroe activo, el que puede cambiar el mundo, imponer las reglas, imaginar y crear nuevas soluciones y escenarios, mientras que los Estados en los que se interviene son representados como las victimas pasivas o como los gobiernos corruptos e ineficientes. En este tipo de narrativas no hay espacios para las historias sobre los esfuerzos de las personas que viven en esos Estados para cambiar sus vidas o las de su comunidad. Su único guión posible es el que les remite a buscar la protección de la comunidad internacional como ente salvador. Cualquier lector o lectora de esta historia tenderá irremediablemente a identificarse con el caballero de la blanca armadura que trae consigo la libertad y la justicia, y se compromete con el mundo mediante sus acciones ${ }^{13}$. Son este tipo de justificaciones y argumentaciones sobre las que posteriormente se asentarán decisiones internacionales sobre la intervención en un determinado país y la forma en la que ésta se llevará a cabo.

En Derecho Internacional tendemos a pensar que una vez cesan los enfrentamientos armados la guerra ha terminado y comienza el periodo de paz inaugurado por un tratado. La construcción de la paz por lo tanto se desliga del conflicto, se construye sobre los daños en forma de reparación. El Derecho Internacional trata en la mayoría de los casos de reparar la guerra, no de construir

que sentían por que las mujeres fuera del ámbito domestico fueran caprichosas o avariciosas. Definieron la vida republicana, moral y civilizada en oposición a ese deseo inculto y retrógrado que identificaron con las mujeres y las personas no blancas» (YounG, Iris Marion: «Vida política y diferencia de grupo: una critica del ideal de ciudadanía universal», en Carme Castells (comp.): Perspectivas feministas en teoría política, Barcelona, Paidós, 1996, p. 104).

12. Charlesworth, Hilary: «International Law: A Discipline of Crisis», Modern Law Review, 65 (2002), p. 387. Sobre las implicaciones del análisis jurídico en Derecho Internacional en Kosovo a la hora de catalogar una situación como intervención humanitaria ver ÁLVAREZ, Natalia: «Uso de la fuerza, ansiedad ética y el derecho a preguntar en Derecho Internacional: Una retrospectiva sobre Kosovo» (en prensa).

13. ORFORD, Anne: "Muscular Humanitarism: Reading the Narratives of New Interventionism», European Journal of International Law, 10:4 (1999), p. 695. 
la paz. Crea comisiones internacionales para listar las violaciones de derechos humanos y juzgar a los culpables, impulsa la restauración del poder legislativo, ejecutivo y judicial, asegura un marco normativo acorde a las exigencias de los Estados liberales y promueve la creación de un ejercito que formalmente se comprometa con respetar los derechos humanos. La guerra y la paz se entienden como dos estados antinómicos y opuestos, no hay relación entre ellos más allá del hecho de que la paz se articula como un estado de evitación de la guerra.

Sin embargo, como nos recuerda Foucault, existe un tipo de discurso que hunde sus raíces en la Edad Media y que preconiza que la ley no nace de la naturaleza, nace de batallas reales, de masacres, de conquistas, del horror que tiene nombres y apellidos. En este escenario, "la paz hace sordamente la guerra hasta en el más mínimo de sus engranajes» porque a lo que nos enfrentamos es al enemigo perpetuo, al sujeto beligerante. La reconciliación o la pacificación sólo es posible en la medida en que haya unos efectivos y verdaderos vencedores ${ }^{14}$. La paz entonces es la antesala de otros conflictos que reproducen la dominación y el reparto de poder al que se ha llegado después de la guerra. El feminismo precisamente trata de romper con la visión de que la paz es un estadio en el que por el mero hecho de que el conflicto ha finalizado las mujeres tienen un lugar, una oportunidad de construir la sociedad de forma diferente. La guerra pone en marcha una serie de mecanismos que enraízan con las concepciones más estereotipadas y discriminantes para las mujeres. Después de una guerra, las mujeres se enfrentan a la difícil tarea de la construcción de la paz, que como la de la guerra, sigue siendo más que nunca una cuestión masculina, una cuestión de poder.

\section{LO QUE EL FEMINISMO NOS ENSEÑA SOBRE LA PAZ}

Una de las primera cuestiones que conviene resaltar es que ni la guerra es una categoría de hombres, ni la paz de las mujeres. Este tipo de dicotomías tiene mucho que ver con una simplificación de la realidad que oscurece otro tipo de análisis. La construcción de la paz ni es una tarea de las mujeres, ni estamos mejor dotadas para ello, ni es algo a lo que nuestra naturaleza nos aboque. Al igual que no estamos predestinadas únicamente a reproducir la raza humana o a cuidar de ella, tampoco nada nos obliga a reparar lo que otros u otras han destruido ${ }^{15}$. La guerra y la paz han sido y siguen siendo apoyadas indistintamente por hombres y mujeres a lo largo de la historia. Lo que nos interesa saber por lo tanto es qué implicaciones tiene la construcción de la paz en el Derecho

14. FoUCAUlt, Michel: Hay que defender la sociedad, Madrid, Akal, 2003, pp. 49-50.

15. Una de las características de la diferenciación público-privado en lo que a la esfera del cuidado se refiere radica en el hecho de que sólo las personas (madres o esposas) que no han sido reconocidas fuera, en ese espacio en el que todo es más vulnerable, pueden darnos algún tipo de cuidado. Así, la única dependencia segura es la de aquella persona que no está expuesta a la lucha de todos contra todos, y que de la misma manera, no es ella misma independiente (BENJAMIN, Jessica: The Bonds of Love, New York, Panteon, 1988, p. 202). 
Internacional para las mujeres y si el feminismo puede darnos alguna pista en esta tarea.

Una de las primeras cuestiones a las que nos aboca el feminismo es a la globalidad. Frente a los análisis parcelados, frente a las categorías jurídicas que se imponen, frente a la doctrina dominante, el feminismo nos invita a entender y acercarnos a la construcción de la paz desde la experiencia de la guerra. Esto significa que en Derecho Internacional no deberíamos pasar por alto el hecho de que cómo se ha hecho la guerra, se hará la paz. Las razones que llevan a la intervención de la comunidad internacional, las que llevan a su inhibición, cómo se justifican estas acciones, los medios que se utilizan, el tipo de intervención que se lleva a cabo o los objetivos de la misma, son datos que configurarán en última instancia las claves de la paz que posteriormente se diseñe. Algunas guerras nos recuerdan amargamente que aunque se nos mencione entre los motivos, nunca permanecemos el suficiente tiempo en las agendas políticas como para generar cambios. En Afganistán la situación de las mujeres tras la guerra contra los taliban permitió que éstas accedieran de forma limitada a escuelas, universidades y puestos de trabajo. Sin embargo, tan pronto como la situación se deterioró y los taliban fueron ganando terreno de nuevo, las mujeres se convirtieron en el primer sujeto a sacrificar para aplacar las iras del fundamentalismo. Human Rights Watch señala que desde 2002 era frecuente que se reclutara a niños en las escuelas como informantes para denunciar comportamientos anti-islámicos de sus compañeras de clase ${ }^{16}$. En este clima de deterioro no ha sido una sorpresa el asesinato de Safiye Amajan, activista por los derechos de las mujeres en Afganistán en septiembre de 2006 a manos de los taliban.

En la guerra en la en estos momentos nos encontramos, la guerra contra el terrorismo, la paz parece lejana y se evidencia ilusorio hablar de construcción de la paz en Derecho Internacional cuando es posible utilizar la fuerza armada contra cualquier Estado acusado de colaborar u auspiciar el terrorismo internacional. En un estado de amenaza constante, en el que el enemigo se encuentra en cualquier lugar, en el que la seguridad se convierte en la máxima prioridad, el feminismo parece que pierde su lugar, asediado por la inmediatez del que no sabe qué se encuentra a la vuelta de la esquina o quién será el siguiente en la lista. Si el terrorismo opera y espera agazapado, si no hay objetivos predeterminados que constituyan las principales líneas de actuación, si el terrorismo trabaja desde la sombra, entonces habrá que enfrentarlo desde el lado más invisible del Estado, desde la represión ${ }^{17}$. En esta lucha entre bandos secretos,

16. HUMAN RIGHTS WATCH: Afganistan: "We want to leave as humans». Repression of women and girls in Western Afganistan, vol 14, $\mathrm{n}^{\circ} 11$ (C), 2002.

17. En este contexto, entiendo la represión como fuerza jurídica de la prohibición (FoUCAULT, Michel: Estrategias de poder, Obras esenciales, vol. II, Barcelona, Paidós, 1999, p. 48). Conviene resaltar que el número de conductas prohibidas y punibles se incrementa cada día en función de la puesta en marcha de nuevas medidas anti-terroristas. En este sentido, y sólo en España, Human Rights Watch en un informe de 2005 alertaba del deterioro de las garantías penales y procesales para los acusados y acusadas de terrorismo. Ver HUMAN RIGHTS WATCH: ¿Sentando ejemplo? Medidas antiterroristas en España, vol 17, n 1 (D), 2005. 
las mujeres quedamos atrapadas, disfrazadas entre estadísticas de víctimas que no diferencian hombres de mujeres. Sin embargo, sabemos por experiencia que estas guerras se sostienen por una maquinaria en la que las mujeres nunca salimos ganando.

La guerra contra el terrorismo también ha hecho que algunas feministas como McKinnon devolvieran al Estado sus propios argumentos. McKinnon asegura que si 3000 personas muertas en los ataques a las torres gemelas son suficientes para iniciar una guerra contra el terrorismo, quizás convendría tener en cuenta que las mujeres que mueren a manos de sus parejas o son agredidas en Estados Unidos sobrepasan anualmente esa cifra. Esta autora defiende que los actos de violencia contra las mujeres son similares al terrorismo, se llevan a cabo por individuos que no son Estados contra un colectivo de mujeres que se encuentra en cualquier lugar del mundo. Aparentemente, Mckinnon no trata de plantearse si la guerra es la respuesta a ambos tipos de terrorismo, pero su pregunta va dirigida a por qué en unos casos se actúa y en otros no ${ }^{18}$. Su argumento muestra que las decisiones en Derecho Internacional que avalan intervenciones armadas se basan en criterios que fácilmente pueden ser contestados con argumentos políticos de igual peso. La propuesta de Mckinnon nos puede parecer exagerada o inadecuada, pero lo cierto es que como argumento ha logrado llevar a la arena pública el debate sobre la violencia contra las mujeres en Estados Unidos.

El feminismo también nos enseña, como señala Enloe, que no es suficiente hablar de militarismo, sino que es importante ser conscientes de cómo gana terreno la militarización en una sociedad, y como ésta se basa sobre una determinada construcción de la masculinidad y la feminidad ${ }^{19}$. El militarismo como ideología preconiza que debido a que la naturaleza humana tiende irremediablemente a los conflictos, las fuerzas armadas son en última instancia las que pueden resolverlos dentro de en un Estado. Por ello, tener enemigos es una condición natural y para paliar esta situación que nos viene dada es necesario establecer relaciones jerárquicas basadas en la efectividad. En tiempos de guerra, las mujeres necesitan ser protegidas y los hombres, por su parte, deben optar por enrolarse en el conflicto armado violento, debido a que no hacerlo implica poner en peligro su propio status como hombres ${ }^{20}$. En consonancia con el militarismo, la militarización es un proceso socio-político que se produce en los llamados tiempos de paz en el que la sociedad se prepara para utilizar la violencia. Estos dos procesos están entrelazados a través de potentes roles de género. La militarización de nuestra sociedad se cimienta sobre una determinada ideología que asigna unos roles a los hombres y otros a las mujeres. Cuando la guerra cesa, estas categorías que han operado durante tanto tiempo para que

18. MACKInNON, Catherine A.: «Women's September 11th: Rethinking the International Law of Conflict», Harvard International Law Journal, 47:1 (2006), pp. 2-31.

19. EnLOE, Cynthia: The curious feminist: Searching for women in a new age of empire, London, University of California Press, 2004, p. 219.

20. Ibíd., p. 219. 
la guerra fuera posible y deseable en la mente de todos los que tomaron parte en ella, siguen funcionando como imaginario posible para la paz. Los tratados de paz no ponen fin a las ideologías, reparten únicamente el poder entre vencedores y vencidos, al mismo tiempo que sientan una nuevas bases jurídicas sobre las que ha de asentarse el Estado. Estas circunstancias no necesariamente ordenan o alteran los estereotipos e ideas sobre las mujeres que alimentaron y sostuvieron la guerra. En muchos casos, las voces que más se oyen siguen siendo las de los hombres, y especialmente las de los militares. Este aspecto es especialmente relevante en lo que concierne al concepto de seguridad, que suele definirse según parámetros que no recogen necesariamente las necesidades de las mujeres. De hecho, suele ser habitual que el tipo de inseguridad que las mujeres experimentan en tiempos de la reconstrucción sea similar al que experimentaban durante la guerra ${ }^{21}$.

Enloe afirma que cuando los hombres vuelven a casa después de una guerra, se producen importantes ajustes personales. En muchas ocasiones el soldado herido física o psicológicamente necesita un apoyo que el gobierno no puede o no está dispuesto a dar. En estos casos, los gobiernos de la post-guerra suelen contar con las mujeres para estas tareas. Las mujeres suelen acusar la presión de tener que afrontar difíciles situaciones personales sin ayuda ni recursos, sobre todo en momentos en los que tras el agotamiento de la guerra se les sigue exigiendo que sigan sosteniendo a sus familias, en consecuencia el número de divorcios aumenta, lo que provoca que las mujeres necesiten empleos para salir adelante económicamente. Todas estas circunstancias se producen cuando los gobiernos se encuentran ante la necesidad de tener que desmovilizar parte de sus tropas y reorganizar un mercado laboral precario tras la guerra, que requiere ciertos ajustes para poder absorber a los antiguos combatientes. Las prioridades están claras. No se concibe que una mujer divorciada y un veterano de guerra puedan competir por el mismo trabajo ${ }^{22}$. A las mujeres, después de las guerras, se les suele pedir que esperen, que sean pacientes, que no es el momento de sacar adelante demandas de género porque el país no está preparado. Enloe nos recuerda que cuando viene el tiempo de la desmovilización de sectores que conformaban el ejército que luchó en una guerra, siempre se desmoviliza primero a determinados grupos étnicos y a las mujeres, el objetivo es claro, se trata de «remasculinizar el ejercito otra vez» y lo antes posible ${ }^{23}$.

En lo relativo a los procesos de paz, las experiencias y voces desde el feminismo son muy diversas. En general, existe una tendencia a sostener que el hecho de que las mujeres participen en los procesos de negociación de la paz facilita que las cuestiones de género se incluyan. Sin embargo, existen ejemplos como el Salvador en el que el hecho de que casi un tercio de los representantes del FMLN (Frente para el Movimiento Nacional Farabundo Martí) en la mesa

21. Ibíd., p. 225.

22. Ibíd., pp. 205-207.

23. Ibíd., p. 212. 
de negociaciones fueran mujeres no trajo como resultado que las cuestiones de género estuvieran más presentes. En mi opinión, no es fácil sostener una postura cerrada y firme para todos los casos pero me inclino a pensar que la cantidad no siempre es sinónimo de calidad ${ }^{24}$. En Afganistán el número de mujeres que accedieron a la conferencia de paz fue muy escaso y cuando hubo que formar gobierno y comenzaron las críticas en relación con la inexistencia de representantes femeninas, en muchos casos se optó por aquellas candidatas con menos experiencia frente a las que habían mostrado una mayor actividad política. La comunidad internacional no ha sido neutral en este terreno. En muchos casos ha priorizado a los interlocutores afganos que hablaban inglés, eran hombres, de entorno urbano y mostraban una total ignorancia frente a las cuestiones de género ${ }^{25}$. En octubre de 2000, el gobierno de Estados Unidos votó a favor de la resolución 1325, junto con la mayoría de los miembros del Consejo de Seguridad, en la que se sostenía que las Naciones Unidas y sus agencias se asegurarían que tanto hombres como mujeres fueran parte integrante de las nuevas instituciones en Irak, al mismo tiempo que se reconocía que los intereses de las mujeres en la construcción de la paz y reconstrucción de la nación debían de ser tomados en cuenta. Sin embargo, cuando el gobierno de Estados Unidos designó el comité para elaborar el borrador de la constitución en Irak eligió solamente a varones ${ }^{26}$.

La reconstrucción es así un escenario en el que se toman multitud de decisiones de género. No se trata solamente de que en los tratados de paz se incluyan disposiciones sobre protección de los derechos de las mujeres, o que el Estado se comprometa a ratificar tratados internacionales que protegen a las mujeres contra la discriminación. Otro tipo de decisiones también cuenta. Por ejemplo ¿Quién recibe la formación para insertarse en el mercado laboral? ¿A quién se contrata? ¿Qué sexualidad se controla en los programas de control de natalidad para reducir la presión sobre el mercado y el acceso a los recursos?27 ¿Se persigue la violencia contra las mujeres? ¿Cómo se aborda la cuestión de la violencia sexual contra las mujeres durante la guerra y después de la guerra? ¿Se consulta a los grupos de mujeres antes de adoptar medidas que les afectan? ¿Qué tipo de consultas se llevan a cabo y con qué procedimientos? Todas estas cuestiones aparentemente irrelevantes desde el punto de vista del Derecho Internacional configuran algunas de las preguntas e intereses básicos que las mujeres tienen después de una guerra. La mayoría de estas disposiciones se insertan jurídicamente en el ámbito de los derechos humanos, pero otras relativas a aspectos

24. Esto es, pocas representantes pueden hacer mucho, y muchas pueden que no lleguen a hacer nada. En cualquier caso, el número de representantes debe también ponerse en relación con el hecho de a quién representan esas mujeres, y si están organizadas y respaldadas por colectivos o grupos de mujeres que se sienten representados por ellas.

25. AZARBAIJANI-MOGHADDAM, Sippi: "Afgani women on the margins of the twenty first century", en Antonio Donini (ed): Nation-Building Unraveled?: Aid, Peace and Justice in Afganistan, Bloomfield, Kumarian Press, 2003, p. 103.

26. ENLOE, Cynthia: Op. cit., p. 301.

27. Ibíd., p. 214. 
tales como cuáles son los sectores de la economía que se van a reactivar si los mayoritariamente masculinos o los femeninos, conforman un espectro amplio y difuso que se sitúa dentro de las decisiones y elecciones políticas que un Estado toma después de una guerra.

La construcción de la paz, la reconstrucción, la post-guerra se mezclan así como términos de referencia en los que los intereses de las mujeres pueden diluirse con mucha facilidad, pero también pueden igualmente desafiarse. Al inicio de este articulo me he referido a Regina McKnight en un contexto que aparentemente nada tiene que ver con la guerra, en un lugar en el que las mujeres no viven asediadas por un conflicto armado. Sin embargo, el control que se ejerce sobre sus vidas y sus cuerpos es similar. El feminismo nos enseña que tanto en la guerra como en la paz, los estereotipos y la discriminación se transmutan en diferentes escenarios. Existen muchas paces que se han construido al margen de los deseos y aspiraciones de las mujeres. Pero nada nos obliga a repetirlas. Podemos optar por transgredirlas, transformarlas, travestirlas de nuevo no como espacios neutrales en el que el género de esconde, sino como lugares plurales en los que las aspiraciones y deseos de las mujeres pueden discutirse, valorarse, desecharse o (re)elaborarse, un lugar en el que no es necesario que nuestros deseos se transformen automáticamente en derechos, en lugar, en definitiva, en el que realmente podamos deponer las armas. 